\title{
AUGER RECOMBINATION IN QUANTUM WELL LASER WITH PARTICIPATION OF ELECTRONS IN WAVEGUIDE REGION
}

\author{
A.A. Karpova ${ }^{1,2}$, D.M. Samosvat ${ }^{2}$, A.G. Zegrya ${ }^{2}$, G.G. Zegrya ${ }^{1,2}$ and V.E. Bugrov ${ }^{1}$ \\ ${ }^{1}$ Saint Petersburg National Research University of Information Technologies, Mechanics and Optics, \\ Kronverksky Pr. 49, St. Petersburg, 197101 Russia \\ ${ }^{2}$ loffe Institute, Politekhnicheskaya 26, St. Petersburg, 194021 Russia
}

Received: May 07, 2018

\begin{abstract}
A new mechanism of nonradiative recombination of nonequilibrium carriers in semiconductor quantum wells is suggested and discussed. For a studied Auger recombination process the energy of localized electron-hole pair is transferred to barrier carriers due to Coulomb interaction. The analysis of the rate and the coefficient of this process is carried out. It is shown, that there exists two processes of thresholdless and quasithreshold types, and thresholdless one is dominant. The coefficient of studied process is a non-monotonous function of quantum well width having maximum in region of narrow quantum wells. Comparison of this process with $\mathrm{CHCC}$ process shows that these two processes of nonradiative recombination are competing in narrow quantum wells, but prevail at different quantum well widths.
\end{abstract}

\section{INTRODUCTION}

Nowadays an actual research field of semiconductor optoelectronics is InGaAsP/InP multiple quantum well (MQW) lasers, because their lasing wavelength is $1.3-1.55$ micrometers and coincides with transparency windows of optical fiber [1-5]. It is known [2], that lasers based on InGaAsP/InP have some shortcomings: the threshold current strongly depends on temperature above the temperature of active region $T>60^{\circ} \mathrm{C}$. Also at high pump levels generation fails. It is assumed, that this effect is caused by carrier and lattice heating [2].

For laser structure with InGaAsP/InP quantum wells considered in this paper the depth of QWs for electrons and holes are $0.08 \mathrm{eV}$ and $0.464 \mathrm{eV}$, respectively. At high excitation levels and high temperatures electrons are partly ejected from the $\mathrm{QW}$ into the waveguide region [1]. Also as shown in [6], that even for structures with deeper QWs part of nonequilibrium carriers is still located in the waveguide region.

In present work a new loss channel in InGaAsP/ InP MQW lasers is under consideration. It affects significantly the threshold characteristics of laser and leads to generation failure at high excitation levels and temperatures. For such Auger recombination the recombination energy of localized electron-hole pair is transferred to barrier carriers due to Coulomb interaction (Fig. 1).

It is known, that the lifetime of electron localized in QW depends on three processes such as radiative recombination, nonradiative Auger recombination of confined electrons and holes $[7,8]$ and nonradiative recombination of confined carriers interacting with electrons in the waveguide region. Thus, the last process should be considered as well if the threshold characteristics of lasers are analyzed. We will use four-band Kane's model [9],

Corresponding author:A.G. Zegrya, e-mail: zegrya@theory.ioffe.ru 
based on 8x8 Hamiltonian [10], which describes in the most accurate way the wave functions and energy spectra of carriers in narrow-gap $A^{\prime \prime \prime} B^{v}$ semiconductors.

The aim of the present work is to perform a detailed analysis of rate and coefficient for the studied process of Auger recombination, the dependence of its coefficient on QW width and a comparison of this process with $\mathrm{CHCC}$ for the model structure [2].

\section{BASIC EQUATIONS}

Because of a small effective mass electron are just partly localized in a narrow QW, while holes are almost completely localized therein. As a result a carrier redistribution, which is equal to charge redistribution, takes place over the active region consisting of QWs and waveguide region. To approximate the electron density in the barrier region a selfconsistent Poisson's equation is used. An additional equation that relates the quasi-Fermi levels for electrons and holes is:

$p_{a w}^{2 D}=n_{a w}^{2 D}+n_{b}^{2 D}$,

where $n_{a w}^{2 D}, p_{a w}^{2 D}$, and $n_{b}^{2 D}$ are 2D carrier concentrations of electrons and holes in QW and barrier region. Final expressions for 2D carrier concentrations [2] are:

$$
\begin{aligned}
& n_{b}^{2 D}=\sqrt{\frac{4}{3 \pi}} N_{c}^{2 D}(T)\left(\frac{T}{E_{B}}\right)^{1 / 4} \times \\
& \left(\int_{0}^{\infty} \frac{\varepsilon^{3 / 2} \mathrm{~d} \varepsilon}{1+\exp \left(\varepsilon+u_{c}-\eta_{n}\right)}-\right. \\
& \left.\int_{0}^{\infty} \frac{\varepsilon^{3 / 2} \mathrm{~d} \varepsilon}{1+\exp \left(\varepsilon+u_{c}+\tilde{\phi}_{0}-\eta_{n}\right)}\right)^{1 / 2}, \\
& n_{O W}^{2 D}=N_{c}^{2 D} \ln \left(\exp \left(\frac{\mu_{n}-\varepsilon_{n}}{T}\right)+1\right), \\
& p_{O W}^{2 D}=\frac{m_{h h}}{m_{c}} N_{c}^{2 D} \ln \left(\exp \left(\frac{\mu_{p}-\varepsilon_{p}}{T}\right)+1\right),
\end{aligned}
$$

where $m_{c}^{\prime}, m_{c^{\prime}}$ and $m_{h h}$, are effective electron masses in barrier region and in $\mathrm{QW}$ and effective heavy hole mass in QW respectively, $N_{c}^{\prime 2 D}=m_{c}^{\prime} T / \pi \hbar^{2}$ and $N_{c}^{2 D}=m_{c} T / \pi \hbar^{2}$ are two-dimensional effective density of states for conduction band in barrier region and in QW, $E_{B}=m_{c}^{\prime} e^{4} / 2 \varepsilon_{s} \hbar^{2}$ is the Bohr energy in barrier region, $u_{c}=\Delta E_{c} / T, \Delta E_{c}$ is conduction band discontinuity and the barrier height for electrons,

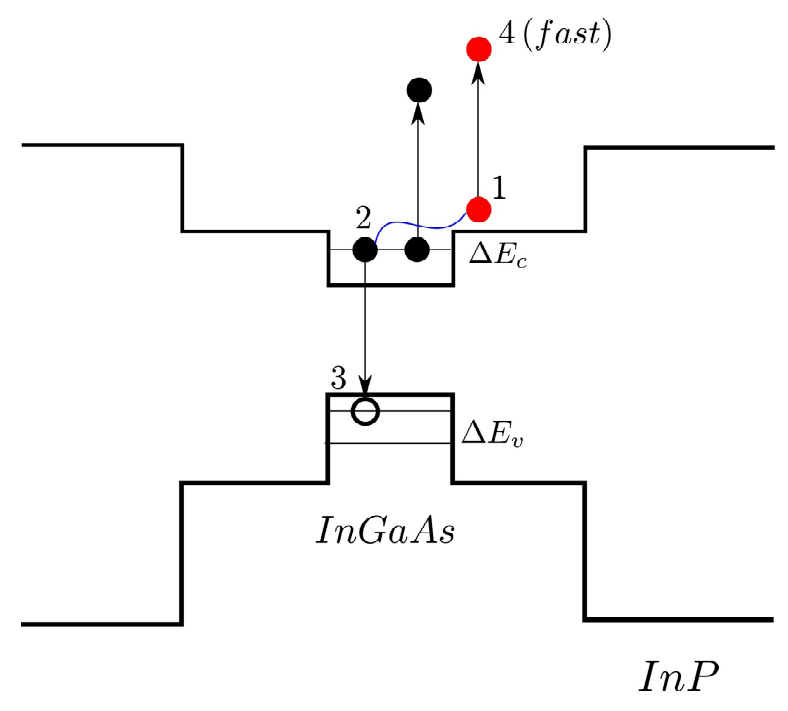

Fig. 1. Two schemes of electron transitions for CHCC-process and the studied AR in QW

$\phi_{n}=\mu_{n} / T, \tilde{\phi}=-e \phi / T, \phi$ is electron potential, $\mu_{n}$ and $\mu_{p}$ are the quasi-Fermi levels for electrons and holes, $\varepsilon_{n}$ and $\varepsilon_{p}$ are quantized energy levels of an electron and a hole in the conduction and valence bands respectively, $T$ is the temperature of electron-hole plasma in terms of energy. The quasi-Fermi levels for electrons and holes are measured from the band edges as well as quantized energy levels of localized carriers.

\section{WAVE FUNCTIONS AND CARRIER STATES IN QW AND BARRIER REGION}

Let us consider a single QW with its width a, located so that $x$-axis is its axis of symmetry (Fig. 2). To find the rate and the coefficient of studied Auger process the wave functions of carriers are needed. For most $A^{\prime \prime \prime} B^{V}$ semiconductors the wave functions of the conduction band are described $\Gamma_{6}^{+}$representation, and the wave functions of the valence band are described by $\Gamma_{7}^{+}$and $\Gamma_{8}^{+}$representations. The equations for the corresponding wave functions can be written in differential form. The following representation of the basis functions is chosen, which seems to be more suitable for the present work [7]:

$$
\begin{aligned}
& |s \uparrow\rangle,|s \downarrow\rangle,|x \uparrow\rangle,|x \downarrow\rangle,|y \uparrow\rangle,|y \downarrow\rangle, \\
& |z \uparrow\rangle,|z \downarrow\rangle,
\end{aligned}
$$

where $|s\rangle,|x\rangle,|y\rangle,|z\rangle$ are $s$ - and $p$-type Bloch functions with angular momentum 0 and 1 respectively. The arrows denote spin directions.

The carrier wave function $\psi$ has following representation: 


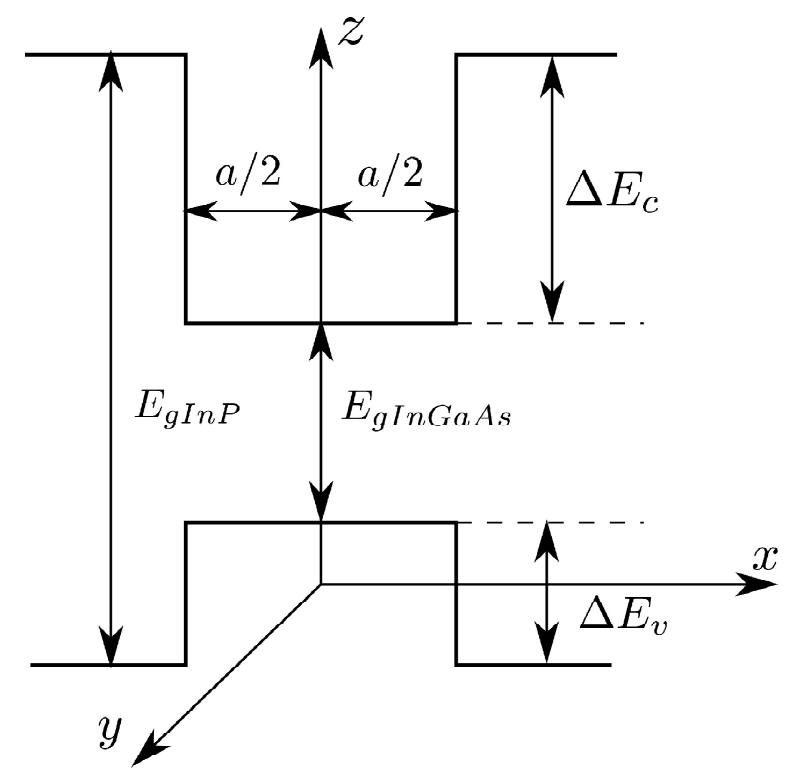

Fig. 2. A schematic representation of a single $Q W$ of InGaAsP/InP heterostructure.

$\psi=\Psi_{s}|s\rangle+\boldsymbol{\Psi}|\boldsymbol{p}\rangle$,

where $\Psi_{\mathrm{s}}$ and $\Psi$ are spinors. Near the $\Gamma$ point the equations for the envelope functions $\Psi_{\mathrm{s}}$ and $\Psi$ in the spherical approximation have a form [7]:

$$
\left\{\begin{array}{l}
\left(E_{c}-E\right) \Psi_{s}-i \hbar \gamma \nabla \boldsymbol{\Psi}=0, \\
\left(E_{v}-\delta-E\right) \boldsymbol{\Psi}-i \hbar \gamma \nabla \Psi_{s}+ \\
\frac{\hbar^{2}}{2 m}\left(\tilde{\gamma}_{1}+4 \tilde{\gamma}_{2}\right) \nabla(\nabla \boldsymbol{\Psi})- \\
\frac{\hbar^{2}}{2 m}\left(\tilde{\gamma}_{1}-2 \tilde{\gamma}_{2}\right)[\nabla[\nabla \boldsymbol{\Psi}]]+i \delta[\boldsymbol{\sigma} \boldsymbol{\Psi}]=0 .
\end{array}\right.
$$

Here $\gamma$ is the Kane matrix element, $\tilde{\gamma}_{1}, \tilde{\gamma}_{2}=\tilde{\gamma}_{3}$ are generalized Luttinger parameters, $\delta=\Delta_{\mathrm{so}} / 3, \Delta_{\mathrm{so}}$ is the spin-orbit splitting constant of $Q W$ semiconductor, $E_{c}$ and $E_{v}$ are the energies of the lower edge of the conduction band and the upper edge of the valence band, $m$ is a free electron mass, $\sigma=\left(\sigma_{x}, \sigma_{y^{\prime}}, \sigma_{z}\right)$ denotes the Pauli matrices.

For reasons of convenience, the energy of the upper edge of the valence band $E_{v}$ is set equal to $\delta$ as it is shown in Fig. 2.

An explicit form of carrier wave functions and expressions determining their boundary conditions, energy spectra and dispersion equations could be found in $[7,8]$. The wave functions of electrons in the barrier region have the same form with wave functions of electrons localized in QWs. The only difference is replacement of quantity $k_{c}$ with quantity $\tilde{k}_{c}=i \kappa_{c}$ for $|x|>a / 2$.

\section{AUGER RECOMBINATION RATE AND COEFFICIENT}

With the account of the antisymmetrized form of the wave functions, the matrix element of the Auger process is the following:

$M_{f i}=M_{l}-M_{\| l}$,

$M_{l}=\left\langle\Psi_{3}\left(\boldsymbol{r}_{1}, v_{1}\right) \Psi_{4}\left(\boldsymbol{r}_{2}, v_{2}\right)\left|\frac{e^{2}}{\kappa_{0}\left|\boldsymbol{r}_{1}-\boldsymbol{r}_{2}\right|}\right| \times\right.$

$\left.\Psi_{1}\left(\boldsymbol{r}_{1}, v_{1}\right) \Psi_{2}\left(\boldsymbol{r}_{2}, v_{2}\right)\right\rangle$,

$M_{\| l}=\left\langle\Psi_{3}\left(\boldsymbol{r}_{1}, v_{1}\right) \Psi_{4}\left(\boldsymbol{r}_{2}, v_{2}\right)\left|\frac{e^{2}}{\varepsilon_{0}\left|\boldsymbol{r}_{1}-\boldsymbol{r}_{2}\right|}\right| \times\right.$

$\left.\Psi_{1}\left(\boldsymbol{r}_{2}, v_{2}\right) \Psi_{2}\left(\boldsymbol{r}_{1}, v_{1}\right)\right\rangle$,

where $\boldsymbol{r}_{1}$ and $\boldsymbol{r}_{2}$ are the carrier coordinates, $v_{1}$ and $v_{2}$ are spin variables, $e$ is a charge of electron, $\varepsilon_{0}$ is the static dielectric constant of the QW semiconductor.

It could be easily shown that the matrix element of direct Coulomb interaction $M_{1}$ is much larger than the matrix element of exchange interaction $M_{\|}: M_{1}>>M_{\|}$.

To estimate the matrix element of investigated Auger process for a transition of an excited particle into continuous spectrum the following approximation could be made: $\Delta E_{c}, \Delta E_{v}<<E_{g}$, what implies $k_{4}^{2}+q^{2}>>k_{1}^{2}$. It means that the total momentum of an excited particle is much larger than that of a localized one. The matrix element could be calculated using the Fourier representation:

$$
\begin{aligned}
& M_{l}=\frac{4 \pi e^{2}}{\kappa_{0}} \int \frac{l_{23}(p) I_{14}(-p)}{p^{2}+q^{2}} \frac{\mathrm{d} p}{2 \pi} \delta_{q 1+q 2-q 3-q 4}, \\
& I_{i j}(p)=\int \Psi_{i}^{*}(x) \Psi_{j}(x) e^{i p x} \mathrm{~d} x, \\
& \delta_{q}= \begin{cases}1, & q=0, \\
0, & q \neq 0 .\end{cases}
\end{aligned}
$$

where $\boldsymbol{q}=\left|\boldsymbol{q}_{1}-\boldsymbol{q}_{2}\right|=\left|\boldsymbol{q}_{3}-\boldsymbol{q}_{2}\right|$ is the momentum transferred in the plane of the quantum well during the Coulomb interaction, $p$ is the transverse transferred momentum. 
The matrix element could be divided into two parts determined by integration areas. The first term contains the multiplier that decays exponentially away from an interface. The second part arises from integration of the wave functions of carriers within the quantum well. As a result, the matrix element could be represented in a following form:

$$
M=\frac{8 \pi e^{2}}{\kappa_{0}}\left(M_{1}+M_{2}\right)
$$

The mechanisms that lead to violation of the momentum conservation law $k_{1}+k_{2} \neq k_{3}+k_{4}$ are different for two terms $M_{1}$ and $M_{2}$. For the first term $M_{1}$ the momentum nonconservation is associated with the scattering of carriers on the interfaces, and this matrix element corresponds to a true threshold-free process. The reason why the conservation law breaks down for $M_{2}$ is that the volume of integration with respect to $x$ is restricted to the $Q W$ region, and carrier momentum has an uncertainty.

Approximation $q<<k_{4}$ and $k_{1}^{2} / k_{4}^{2}<<1$ and use of the expressions for the carrier wave functions at an interface gives for $M_{1}$ and $M_{2}$ in case of $v_{c}= \pm v_{h}$ :

$$
\begin{aligned}
& M_{1}=\frac{1}{2\left(q^{2}+k_{h}^{2}\right)} \frac{i \hbar \gamma k_{c}^{2} k_{h} q_{h}}{E_{g} \sqrt{k_{c}^{2}+\kappa_{c}^{2}}} \times \\
& \left.\frac{\hbar^{2} \gamma^{2}\left(\Delta E_{c}+\Delta E_{v}\right)}{4 E_{g}^{3}} A_{1} H_{2} \Psi_{4} \Psi_{1}^{*}\right|_{\frac{a}{2}-0}-
\end{aligned}
$$

$$
\left.\frac{2 i \hbar \gamma k_{c}^{2} \kappa_{c} k_{h} q_{h}}{E_{g} \sqrt{k_{c}^{2}+\kappa_{c}^{2}}} \frac{1}{k_{4}^{4}} A_{1} H_{2} \Psi_{4}^{\prime} \Psi_{1}^{*}\right|_{\frac{a}{2}-0},
$$

$$
\begin{aligned}
& M_{2} \approx \frac{1}{8\left(q_{h}^{2}+k_{h}^{2}\right)} \frac{i \hbar \gamma}{E_{g}} A_{c 1} A_{h 2} A_{1} B_{1} \times \\
& \frac{\sin \left(k_{4}-k_{1}-k_{c}-k_{h}\right) a / 2}{k_{4}-k_{1}-k_{c}-k_{h}}\left(q_{h} k_{c}+q_{c} k_{h}\right) .
\end{aligned}
$$

To calculate the AR rate, the probabilities of Auger transition should be summed over all initial and ûnal states of carriers with appropriate weights-occupation numbers. The result of averaging the matrix elements over the initial states and summation over the final states has a form:

$$
\begin{aligned}
& \left\langle M^{2}\right\rangle=\left\langle\sum_{v_{3}, v_{4}}\left|M_{t i}\right|^{2}\right\rangle=\sum_{i}\left(\left|M_{l}^{(i)}\right|^{2}+\left|M_{\|}^{(i)}\right|^{2}-\right. \\
& \left.\operatorname{Re}\left[M_{l}^{(i)^{*}} M_{\|}^{(i)}\right]\right) \approx 4\left|M_{l}\right|^{2},
\end{aligned}
$$

where $M_{1}^{(i)}$ and $M_{1}^{(i)}$ are transition matrix elements with fixed spin states of carriers.
The Auger recombination rate is given by:

$$
\begin{aligned}
& G=\frac{2 \pi}{\hbar} \sum_{\boldsymbol{k}_{1}, \boldsymbol{k}_{2}, \boldsymbol{k}_{3}, \boldsymbol{k}_{4}}\left\langle M^{2}\right\rangle f_{1} f_{2} \times \\
& \left(1-f_{3}\right)\left(1-f_{4}\right) \delta\left(E_{3}+E_{4}-E_{1}-E_{2}\right),
\end{aligned}
$$

where $f_{1}$ and $f_{2}$ are the Fermi distribution functions of the carriers in the initial state, $f_{3}$ and $f_{4}$ are the Fermi distribution functions of the carriers in the final state.

The Auger recombination coefficient $C$ is related to the rate $G$ by the expression:

$$
\tilde{G}=C n_{b} n_{o w}^{2 D} p_{o w}^{2 D}
$$

where $\tilde{G}=G / S$ and $S$ is a normalization area.

To calculate the AR several additional approximations can be made for simplicity:

$$
q_{1} \approx \frac{\sqrt{2 m_{c}^{\prime} T}}{\hbar}, q_{2} \approx \frac{\sqrt{2 m_{c} T}}{\hbar}<<q_{3}, q_{4} .
$$

Integrating (18) over $q_{4}$, then over $k_{4}$ and using energy conservation law and the conservation law of longitudinal components of carrier momenta we obtain the final expression for AR rate:

$$
\begin{aligned}
& \tilde{G} \approx n_{Q w}^{2 D} p_{a w}^{2 D} \frac{2 \pi}{\hbar} \int \frac{\mathrm{d} k_{1} d^{2} q_{1}}{(2 \pi)^{6}} \times \\
& f_{1}\left\langle|\tilde{M}|^{2} \frac{m_{c}}{\hbar^{2}} \frac{1}{k_{4}\left(q_{h}\right)}\right\rangle .
\end{aligned}
$$

Angular brackets in (20) denote averaging over the heavy hole distribution function. In case of a Boltzmann distribution, which is a common case for holes, the averaging looks like

$$
\begin{aligned}
& \left\langle f\left(q_{h}, k_{h}\right)\right\rangle=\frac{1}{Z} \sum_{n} \int_{0}^{\infty} q_{h} f\left(q_{h}, k_{h n}\right) \times \\
& \exp \left(-\frac{\left(k_{h n}^{2}+q_{h}^{2}\right)}{q_{T}^{2}}\right) \mathrm{d} q_{h},
\end{aligned}
$$

where

$$
Z=\frac{q_{T}^{2}}{2} \sum_{n} \exp \left(-\frac{k_{h n}^{2}}{q_{T}^{2}}\right)
$$

is a statistical sum,

$$
q_{T}=\frac{\sqrt{2 m_{h h} T}}{\hbar} .
$$


Integrating (20) over

$q_{1}=\frac{\sqrt{2 m_{c}^{\prime} T}}{\hbar}$

and considering the AR matrix element to be independent of this variable a following expression for coefficient might be obtained:

$C \approx \frac{2 \pi}{\hbar} \frac{1}{(2 \pi)^{3}} \frac{1}{L}\left\langle|\tilde{M}|^{2} \frac{m_{c}}{\hbar^{2}} \frac{1}{k_{4}\left(q_{h}\right)}\right\rangle$.

Here

$$
L=\sqrt{\frac{\pi}{4}} a_{B}\left(\frac{E_{B}}{T}\right)^{1 / 4}
$$

is a characteristic length associated with electron spreading in the subbarrier regions, $a_{B}=\varepsilon_{s} \hbar^{2} / m_{c} e^{2}$ is the Bohr radius in barrier region.

Let us estimate the coefficient of the studied AR. The coefficient $C_{2}$ for quasithreshold AR process looks like

$$
\begin{aligned}
& C_{2} \approx \frac{E_{B}}{\hbar} \frac{8 \lambda_{g}^{2}}{L a\left(a+\frac{2}{\kappa_{c}}\right)}\left\langle\frac{\left(q_{h} k_{c}+q_{c} k_{h}\right)^{2}}{\left(q_{h}^{2}+k_{h}^{2}\right)^{3}} \times\right. \\
& \left.\frac{\sin ^{2}\left(k_{f}-k_{1}-k_{c}-k_{h}\right) a / 2}{\left(k_{f}-k_{1}-k_{c}-k_{h}\right)^{2}} \frac{1}{k_{4}\left(q_{h}\right)}\right\rangle,
\end{aligned}
$$

where

$$
\lambda_{g}=\frac{\hbar}{\sqrt{2 m_{c} E_{g}}} .
$$

The coefficient $C_{1}$ for threshold AR process could be represented in a form:

$$
\begin{aligned}
& C_{1} \approx 4 \pi \frac{E_{B}}{\hbar} \frac{\lambda_{g}^{6}}{L a\left(a+\frac{2}{\kappa_{c}}\right)}\left(\frac{\Delta E_{c}+\Delta E_{v}}{E_{g}}\right)^{2} \times \\
& \left\langle\frac{\left(k_{c}^{2} k_{h} q_{h}\right)}{\left(q_{h}^{2}+k_{h}^{2}\right)\left(k_{c}^{2}+\kappa_{c}^{2}\right)} \frac{1}{k_{4}\left(q_{h}\right)}\right\rangle .
\end{aligned}
$$

\section{RESULTS AND DISCUSSION}

Fig. 3 shows the dependences of $A R$ coefficient $C^{B}$ for the studied process and its components $C_{1}{ }^{B}$ and $C_{2}{ }^{B}$ for threshold and quasithreshold processes on the quantum well width $a$ at $T=20^{\circ} \mathrm{C}$. It can be clearly seen, that dependences $C^{B}$ and $C_{1}{ }^{B}$ reach

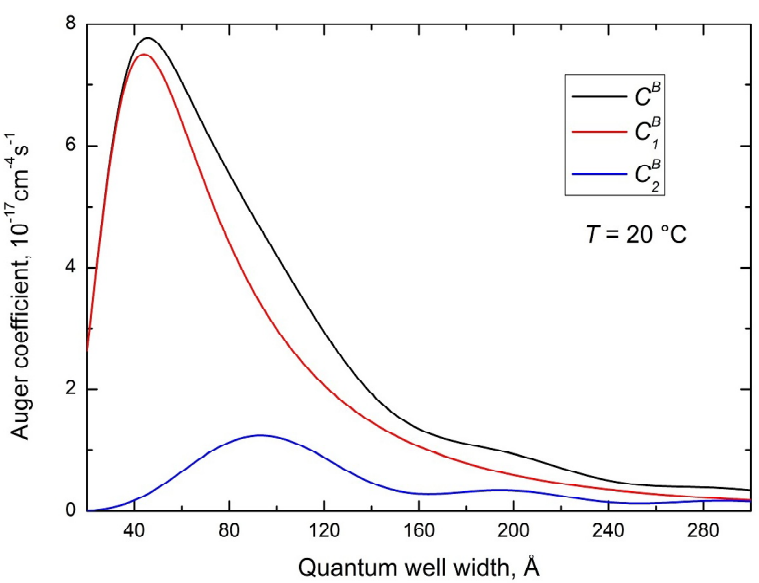

Fig. 3. Dependences of $C^{B}, C_{1}{ }^{B}$, and $C_{2}{ }^{B}$ on $\mathrm{QW}$ width $a$.

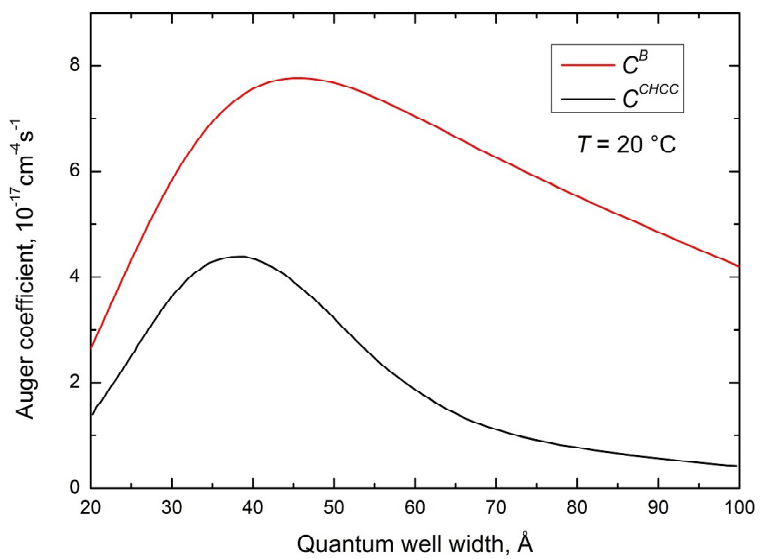

Fig. 4. Dependences of $C^{\mathrm{CHCC}}$ and $C^{B}$ on $\mathrm{QW}$ width $a$.

the peaks at the same $Q W$ width $a \approx 45 \AA$. The dependence for $C_{2}{ }^{B}$ on $\mathrm{QW}$ width maxima at $\mathrm{QW}$ widths $a \approx 94 \AA$ and $a \approx 195 \AA$. One may notice, that the relative contribution of threshold process corresponding to $C_{1}{ }^{B}$ significantly exceeds the contribution of quasithreshold process associated with $C_{2}{ }^{B}$.

Fig. 4 shows the dependences of coefficients $C^{\mathrm{CHCC}}$ and $C^{B}$ on quantum well width a for $\mathrm{CHCC}$ process of Auger recombination and the studied process respectively. According to Fig. 4, two coefficients reach their maximum values at different $\mathrm{QW}$ widths. Thus, the coefficient $C^{\mathrm{CHCC}}$ has a maximum at $a \approx 40 \AA$ and the dependence of coefficient $C^{B}$ on QW width has a peak at $a \approx 45 \AA$.

\section{CONCLUSION}

In this paper a new process of Auger recombination is suggested and discussed. For this process the recombination energy of localized electron and hole is transferred to the carrier in the waveguide region. It is shown, that in InGaAsP/InP MQW lasers the studied process competes with known $\mathrm{CHCC}$ proc- 
ess and might strongly affect the lifetime of nonequilibrium carriers in quantum wells and threshold current density.

\section{REFERENCES}

[1] G.G. Zegrya // Semiconductors 35 (2001) 1001.

[2] L.V. Asryan // Semicond. Sci. Technol. 15 (2000) 1131.

[3] S. Seki // IEEE journal of quantum electronics 32 (1996) 1478.

[4] J.D. Evans // IEEE Journal of Selected Topics in Quantum Electronics 1 (1995) 275.
[5] S.J. Sweeney // IEEE Photonics Technology Letters 10 (1998) 1076.

[6] Z.N. Sokolova, I.S. Tarasov and L.V. Asryan // Semiconductors 45 (2011) 1494.

[7] G.G. Zegrya and A.S. Polkovnikov // JETP 86 (1998) 815.

[8] A.S. Polkovnikov and G.G. Zegrya // Phys. Rev. B58 (1998) 4039.

[9] E.O. Kane // J. Phys. Chem. Solids 1 (1956) 82.

[10] P.C. Sercel and K.J. Vahala // Phys. Rev. B 42 (1990) 3690. 